\title{
Analysis on Complex Target Electromagnetic Scattering Features based on Improved Finite Element Boundary Integral Method
}

\author{
An Xu
}

Jingdezhen ceramic university school of management and economy, Jingdezhen, 334000, China.

Keywords: Finite element boundary Integral; Ray-propagation fast multipole; Fast far-field approximation; Electromagnetic scattering

\begin{abstract}
Computational electromagnetic has been more and more mature after development for dozens of years. Domestic and foreign scholars have proposed many methods especially when it is used for solving 3D complex target numerical value questions. Traditional methods include moment method, finite element method, subsequent finite element boundary integral method, and volume-surface integral method, etc. In this paper, ray-propagation fast multipole method and fast far-field approximation methods are applied in a finite element boundary integral method in order to analyse electromagnetic scattering specific issues of 3D targets more rap-idly and efficiently. Improved finite element boundary integral method is characterised by reduced computing time and lowered memory demand compared with the original simple use of MLFMM.
\end{abstract}

\section{Introduction}

Some acceleration methods have developed with the development of numerical value method, such as fast multipole, MLFMM, H matrix method, low rank matrix compression method, etc. (Umashankar, 1986; Yla-Oijala, 2005). MLFMM treatment of far-field calculation can greatly reduce the memory requirments of the calculation. MLFMM accelerates the matrix vector multiplication, and the calculation time can be reduced. Finite element method has its own defects. When it is used for analysing an open domain problem, it is necessary to add an absorbing boundary as a boundary condition. Therefore, the discrete space for finite element method will be enlarged, therefore the calculated matrix elements will be expanded, the calculation unknown quantity will be improved greatly, and the memory consumed by the calculation will be greatly improved (Reddy, 2015; Wang, 2010).

However, the moment method is regarded as an integral equation numerical value method, which is very effective for solving open domain surface problems. Since it is only necessary to discrete object surface, the calculated unknown quantity will be less (Yang Z, T, 2015; Xu G, et al. 2015). Therefore, the moment method and finite element method are combined together in this paper. The combined method is called the finite element boundary integral method (hereinafter referred to FEBI). When FEBI is used, the MLFMM technology used for the moment method in the boundary integral part can not only reduce memory for calculation, but it can also accelerate the calculation speed and reduce the calculation time.

\section{Finite element boundary integral method and RCS}

\subsection{Finite element boundary integral method}

The finite element boundary integral method is discussed in this paper. The finite element boundary integral method in the boundary integral part is a moment method, therefore the basic principle of the moment method is firstly given in the paper.

The mathematics processing process of the moment method in the electromagnetic calculation is as follows: firstly, a suitable electromagnetic field integral equation is deduced, the integral equation is continuous. The integral equation is converted into a matrix equation through a discrete process of a primary function and test process of a weight function. The matrix equation is then obtained, the induction electromagnetic flow distribution is calculated. An iteration method is used in the paper for the solution. Finally, post-treatment is implemented. According to the induction electromagnetic flow 
distribution, the required parameters are calculated, such as circuit $S$ parameters, antenna radiation field distribution, scatterer RCSRCS, etc. (Ji, Yun, 2000; Ren Y, 2016). Next, the moment method solution process will be introduced.

Operator equation (continuous) can be written into the following form:

$$
L(f)=h
$$

where $\mathrm{L}$ refers to the linear operator, which can be differential, integral or a combination of differential and integral; $\mathrm{h}$ refers to a known function, and $\mathrm{f}$ refers to a to-be-solved unknown function.

Primary function expansion: it can be expressed as a linear combination of a group of primary functions $\left\{f_{1}, f_{2}, f_{3} \ldots\right\}$

$$
f=\sum_{n=1}^{N} \alpha_{n} f_{n}
$$

Equation (2) is substituted into (1) to obtain the following:

$$
\sum_{n=1}^{N} \alpha_{n} L\left(f_{n}\right)=g
$$

Weight function test: one group of weight functions $\left\{w_{1}, w_{2}, w_{3}, \ldots\right\}$ is selected, each weight function and Equation (3) are regarded as inner product:

$$
\sum_{n=1}^{N} \alpha_{n}\left\langle w_{m}, L f_{n}\right\rangle=\left\langle w_{m}, g\right\rangle \quad m=1,2,3, \ldots, N
$$

It is written into matrix equation

$$
[\mathrm{Z}]\{\alpha\}=\{G\}
$$

The matrix equation solution is given by:

$$
\{\alpha\}=[\mathrm{Z}]^{-1}\{G\}
$$

The moment method has the following solution keys aimed at target electromagnetic scattering problems: 1) How to select a primary function and a trial function; 2) singular point treatment technology; 3) Relationship between integral equation form and discrete matrix form; 4) fast solution technology of the moment method discrete matrix equation.

The primary function selected in the paper is the Rao-Wilton-Glisson primary function (hereinafter referred to as RWG primary function). It is a planer primary function, the primary function was proposed by S. M. Rao, D. R. Wilton and A. W. Glisson in 1982, which is widely applied in triangular networks.

In the boundary integral part, the following electric field integral equation and magnetic field integral equation will be used in the paper:

$$
\begin{aligned}
& \mathbf{E}^{i n c}(\mathbf{r})=\frac{1}{2} \mathbf{E}(\mathbf{r})+\mathbf{L}\left(\overline{\mathbf{J}}_{\mathrm{s}}\right)-\mathbf{K}\left(\mathbf{M}_{\mathrm{s}}\right) \\
& \overline{\mathbf{H}}^{i n c}(\mathbf{r})=\frac{1}{2} \overline{\mathbf{H}}(\mathbf{r})+L\left(\mathbf{M}_{\mathrm{s}}\right)+K\left(\overline{\mathbf{J}}_{\mathrm{s}}\right)
\end{aligned}
$$

Equations (7) and (8):

$$
\begin{aligned}
& \overline{\mathbf{J}}_{\mathbf{s}}=\eta \hat{n} \times \mathbf{H} \\
& \mathbf{M}_{s}=\mathbf{E} \times \hat{n} \\
& \oiint_{S} \mathbf{X}\left(\mathbf{r}^{\prime}\right) G_{0}\left(\mathbf{r}, \mathbf{r}^{\prime}\right) \mathrm{d} S^{\prime}+\oiint_{S} \frac{j}{k_{0}} \nabla^{\prime} \cdot \mathbf{X}\left(\mathbf{r}^{\prime}\right) \nabla G_{0}\left(\mathbf{r}, \mathbf{r}^{\prime}\right) \mathrm{d} S^{\prime} \\
& \mathbf{K}(\mathbf{X})=\oiint_{S} \mathbf{X}\left(\mathbf{r}^{\prime}\right) \times \nabla G_{0}\left(\mathbf{r}, \mathbf{r}^{\prime}\right) \mathrm{d} S^{\prime}
\end{aligned}
$$

When the moment method is used for solving the Electric Field Integral Equation (EFIE), the surface tangential component of the object meets the following integral equation:

$$
\left.\boldsymbol{E}^{(i)}(\boldsymbol{r})\right|_{\mathrm{tan}}=\left.j \omega \mu \int_{\sum^{S-r}}\left[\boldsymbol{J}\left(\boldsymbol{r}^{\prime}\right)+\frac{1}{k^{2}} \nabla\left(\nabla^{\prime} \cdot \boldsymbol{J}\left(\boldsymbol{r}^{\prime}\right)\right)\right] G\left(\boldsymbol{r}, \boldsymbol{r}^{\prime}\right) d \boldsymbol{S}^{\prime}\right|_{\mathrm{tan}}
$$


The RWG primary function is used in the paper for discretion of the above integral equation discrete for obtaining resistance matrix as follows:

$$
\begin{aligned}
Z_{m n} & =j k Z \int_{S} \int_{S^{\prime}}\left[\boldsymbol{f}_{m}(\boldsymbol{r}) \cdot \boldsymbol{f}_{n}\left(\boldsymbol{r}^{\prime}\right)-\frac{1}{k^{2}} \nabla_{S} \cdot \boldsymbol{f}_{m}(\boldsymbol{r}) \nabla_{S}^{\prime} \cdot \boldsymbol{f}_{n}\left(\boldsymbol{r}^{\prime}\right)\right] G\left(\boldsymbol{r}, \boldsymbol{r}^{\prime}\right) d \boldsymbol{S}^{\prime} d \boldsymbol{S} \\
& =j k Z \int_{S} \int_{S^{\prime}}\left[\left( \pm \frac{l_{m}}{2 A_{m}^{ \pm}} \boldsymbol{\rho}_{m}^{ \pm}\right) \cdot\left( \pm \frac{l_{n}}{2 A_{n}^{ \pm}} \boldsymbol{\rho}_{n}^{ \pm}\right)-\frac{1}{k^{2}}\left( \pm \frac{l_{m}}{A_{m}^{ \pm}}\right)\left( \pm \frac{l_{n}}{A_{n}^{ \pm}}\right)\right] \frac{e^{-j k R}}{4 \pi R} d \boldsymbol{S}^{\prime} d \boldsymbol{S}
\end{aligned}
$$

In the application process of the moment method, there are different combination modes of the electric field integral equation and the magnetic field integral equation, respectively including $T E N H 、 T E T H 、 N E N H 、 N E T H$, next two difference forms of electric field integral equation and magnetic field integral equation are given in the paper.

Electric field integral equation:

$$
\begin{aligned}
& \hat{t} \cdot \mathbf{E}^{i n c}(\mathbf{r})=\hat{t} \cdot\left[\frac{1}{2} \mathbf{E}(\mathbf{r})+\mathbf{L}\left(\overline{\mathbf{J}}_{\mathrm{s}}\right)-\mathbf{K}\left(\mathbf{M}_{\mathbf{s}}\right)\right] \\
& \hat{n} \times \mathbf{E}^{i n c}(\mathbf{r})=\hat{n} \times\left[\frac{1}{2} \mathbf{E}(\mathbf{r})+\mathbf{L}\left(\overline{\mathbf{J}}_{\mathbf{s}}\right)-\mathbf{K}\left(\mathbf{M}_{\mathbf{s}}\right)\right] \\
& \hat{t} \cdot \overline{\mathbf{H}}^{i n c}(\mathbf{r})=\hat{t} \cdot\left[\frac{1}{2} \overline{\mathbf{H}}(\mathbf{r})+L\left(\mathbf{M}_{\mathbf{s}}\right)+K\left(\overline{\mathbf{J}}_{\mathbf{s}}\right)\right] \\
& \hat{n} \times \overline{\mathbf{H}}^{i n c}(\mathbf{r})=\hat{n} \times\left[\frac{1}{2} \overline{\mathbf{H}}(\mathbf{r})+L\left(\mathbf{M}_{\mathrm{s}}\right)+K\left(\overline{\mathbf{J}}_{\mathrm{s}}\right)\right]
\end{aligned}
$$

The above equation sets are combined for obtaining the matrix form:

$$
\left[\begin{array}{ll}
P & Q
\end{array}\right]\left\{\begin{array}{l}
E^{s} \\
\bar{J}^{s}
\end{array}\right\}=\{b\}
$$

Matrix $P$ and $Q$ obtained from the moment method are full rank matrixes. The calculation internal memory will be highly consumed during direct solution, calculation speed will be reduced. In the paper the MLFMM method is used on the basis of the moment method in order to save the calculation's internal memory and accelerate the matrix's calculation.

In the volume part of the 3D target, the traditional finite element method is adopted in the paper. The finite element method equation is given by:

$$
\begin{aligned}
& \nabla \times\left(\frac{1}{\mu_{r}} \nabla \times \mathbf{E}\right)-k_{0}^{2} \varepsilon_{r} \mathbf{E}=0 \quad r \in V \\
& \hat{n} \times\left(\frac{1}{\mu_{r}} \nabla \times \mathbf{E}\right)=-j k_{0} \hat{n} \times \overline{\mathbf{H}} \text { on the surface of the media; } \hat{n} \times \mathbf{E}(\mathbf{r})=0 \text { on the surface of metal. }
\end{aligned}
$$

Equation (20) is varied for obtaining the following:

$$
F(\mathbf{E})=\frac{1}{2 j k_{0}} \iiint_{v}\left[\frac{1}{\mu_{r}}(\nabla \times \mathbf{E}) \cdot(\nabla \times \mathbf{E})-k_{0}^{2} \varepsilon_{r} \mathbf{E} \cdot \mathbf{E}\right] d V+\eta \oiint \oiint_{S}(\mathbf{E} \times \mathbf{H}) \cdot \hat{n} d S
$$

In Equation (21), the magnetic field $\mathbf{H}$ is only available on the surface of the target, therefore Equation (21) can be converted into the following:

$$
F(\mathbf{E})=\frac{1}{2 j k_{0}} \iiint_{v}\left[\frac{1}{\mu_{r}}(\nabla \times \mathbf{E}) \cdot(\nabla \times \mathbf{E})-k_{0}^{2} \varepsilon_{r} \mathbf{E} \cdot \mathbf{E}\right] d V-\oiint_{S} \mathbf{E} \cdot \overline{\mathbf{J}}_{s} d S
$$

In Equation (22), $\overline{\mathbf{J}}=\eta \mathbf{J}, \mathbf{E}$ and $\overline{\mathbf{J}}_{s}$ are expanded to obtain the following:

$$
\mathbf{E}=\sum_{n=1}^{M} \mathbf{W}_{n} E_{n} \text { and } \mathbf{J}_{s}=\sum_{n=1}^{N_{s}} \bar{J}_{n} \mathbf{f}_{n}
$$

Equation (23) functional equation is varied for obtaining the following:

$$
\left[\begin{array}{lll}
K_{I I} & K_{I S} & 0 \\
K_{S I} & K_{S S} & B
\end{array}\right]\left\{\begin{array}{l}
E_{I} \\
E_{S} \\
\bar{J}_{s}
\end{array}\right\}=\left\{\begin{array}{l}
0 \\
0
\end{array}\right\}
$$


wherein,

$$
\begin{aligned}
& K_{m n}=\sum_{e} K_{i_{m} j_{n}}^{e} \\
& K_{i_{m} j_{n}}^{e}=\frac{1}{j k_{0}} \iiint_{V}\left[\frac{1}{\mu_{r}^{e}}\left(\nabla \times \mathbf{W}_{i}^{e}\right) \cdot\left(\nabla \times \mathbf{W}_{j}^{e}\right)-k_{0}^{2} \varepsilon_{r}^{e} \mathbf{W}_{i}^{e} \cdot \mathbf{W}_{j}^{e}\right] d V \\
& B_{m n}=-\int_{S} \mathbf{W}_{m} \cdot \mathbf{f} n d S=-\int_{S} \sum_{e} \mathbf{w}_{i_{m}} \cdot \mathbf{f}_{n} d S
\end{aligned}
$$

In Equations (25) and (27), $i_{m}$ represents that local number of global edge in unit $e$ is $i, j_{n}$ represents that local number of global edge $n$ in unit $e$ is $j$. The above-mentioned matrix $K 、 B$ belongs to sparse matrix. When FEBI is used for numerical value analysis and calculation, and a sparse memory is adopted for $K 、 B$ storage in the paper.

When the finite element boundary integral method is used, Equation (19) and Equation (24) are combined for the solution. It can be divided into a vipassana equation, appearance equation and mixed equation according to different solution forms. The forms of the three equations in are given as follows: The ipassana equation:

$$
\left[\begin{array}{cc}
K & B^{\prime} \\
P^{\prime} & Q
\end{array}\right]\left\{\begin{array}{l}
E \\
J_{S}
\end{array}\right\}=\left\{\begin{array}{l}
0 \\
b
\end{array}\right\}
$$

Equation (28) is solved, $J_{s}$ is calculated first:

$$
\left[Q-P^{\prime} K^{-1} B^{\prime}\right]\left\{\bar{J}_{s}\right\}=\{b\}
$$

$E$ is then obtained:

$$
\{E\}=-\left[K^{-1} B^{\prime}\right]\left\{\bar{J}_{s}\right\}
$$

When the vipassana equation is used for solving the matrix, the sparse matrix $K$ produced in the part of the finite element should be solved inversely. The consumed memory is relatively less.

Appearance equation:

Equation (19) can express $\bar{J}_{s}$ as follows:

$$
\bar{J}_{s}=Q^{-1}\left(b-P E^{s}\right)
$$

It is added into equation (24):

$\left[\begin{array}{cc}K_{I I} & K_{I S} \\ K_{S I} & K_{S S}-B Q^{-1} P\end{array}\right]\left\{\begin{array}{l}E_{I} \\ E_{S}\end{array}\right\}=\left\{\begin{array}{c}0 \\ -B Q^{-1} b\end{array}\right\}$

$\bar{J}_{s}$ is obtained:

$$
\mathrm{J}_{s}=Q^{-1} b-Q^{-1} P E_{s}
$$

When the appearance equation is used for solving the matrix, matrix $Q$ produced by the moment method should be inversely solved. $Q$ is a full rank matrix, and a lot of memory will be consumed during this inverse solution.

Mixed equation:

$$
\left[\begin{array}{ccc}
K_{I I} & K_{I S} & 0 \\
K_{S I} & K_{S S} & B \\
0 & P & Q
\end{array}\right]\left\{\begin{array}{l}
E_{I} \\
E_{S} \\
\bar{J}_{s}
\end{array}\right\}=\left\{\begin{array}{l}
0 \\
0 \\
b
\end{array}\right\}
$$

The mixed equation refers to the fact that the matrix is obtained using a finite element method which is combined with a matrix obtained from a moment method. The mode can lead to a worse matrix form due to the poor condition. Therefore, when iteration is used for the solution, convergence is slower, and the calculation time is relatively longer. 


\subsection{Radar Cross Section}

Radar Cross Section (hereinafter referred to as RCS) is an equivalent area. The ratio b discretion power of the target to the receiving direction within unit solid angle and the plane wave power density projected to the target from the given direction is $4 \pi$ times (Patel, 2015).

Targets with different properties, shapes and distributions have different radiation efficiency values. In this paper, the effective radiation area is equivalent to the cross section area of the reflector which has the same nature in all directions. It is called the radar cross section area of the target. It is written into $\sigma$. The expression equation is shown as follows:

$$
\sigma=4 \pi A^{2} / \lambda^{2}
$$

where $A$ refers to the irradiated area, $\lambda$ refers to the working wavelength (Graglia, 2015).

\section{Analysis OF complex target electromagnetic scattering features based on AN improved finite element boundary integral method}

Ray-Propagation Fast Multipole method (RPFMA) and FAst Far-Field Approximation method (FAFFA) are methods based on the MLFMM. The fast multipole can reduce demand for calculation memory compared with the moment method since it is not necessary to store far-field elements from the calculation when the fast multipole is used for the far-field calculation. In addition, the fast multipole can accelerate the matrix vector multiplication and reduce the calculation time of the procedure. In this section, ray-propagation fast multipole method and fast far-field approximation method are combined and applied in the boundary integral part of the finite element boundary integral method, since the MLFMM method is adopted for the boundary integral part of the finite element boundary method. After the above two methods are used, the procedure can obtain higher efficiency, which not only can save memory for calculation, but also can accelerate the procedure calculation time.

\subsection{Ray-propagation fast multipole method}

It is firstly assumed that there are two points $r_{i}$ and $r_{j}$ in the calculation area. Then, it is assumed that $r_{i}$ is the field point in the mth observation group. However, $r_{j}$ is the origin of the nth source field group. Finally, $r_{m}$ and $r_{n}$ respectively represent the centres of the observation group and the origin field group. Therefore, the vector from field point to origin can be expressed as $r_{i j}=r_{i}-r_{j}=r_{i m}+r_{m n}+r_{n j}$. If group $\mathrm{m}$ and group $\mathrm{n}$ are not overlapped or adjacent, $r_{i m}+r_{n j}<r_{m n}$ can be obtained, therefore, the 3D scalar quantity green equation can be expanded as follows:

$$
\frac{e^{i k\left|r_{i}-r_{j}\right|}}{r_{i}-r_{j}}=\frac{i k}{4 \pi} \int_{S_{E}} d^{2} \hat{k} e^{i k \hat{k} \cdot\left(r_{i m}+r_{n j}\right)} \alpha_{m n}\left(\hat{k} \cdot \hat{r}_{m n}\right)
$$

The integral in the above Equation (36) is defined in unit ball $S_{E}, \alpha_{m n}$ is the transfer factor of the two groups, which is defined as follows:

$$
\alpha_{m n}\left(\hat{k} \bullet \hat{r}_{m n}\right)=\sum_{j=0}^{L} i^{j}(2 j+1) h_{j}^{(1)}\left(k r_{m n}\right) P_{j}\left(\hat{k}_{\bullet} \hat{r}_{m n}\right)
$$

In Equation (37), $L$ is the truncation item quantity, namely multipole modulus. In addition, $L=k D+\beta(k D)^{1 / 3}$. When low precision is required, $\beta=1.9$, when high precision is required, $\beta=2.9$; $h_{j}^{(1)}(\bullet)$ belongs to the first category of a Bessel function; $P_{j}(\bullet)$ refers to a Legendre polynomial expansion. Here, $D$ refers to group size, $r_{m n}$ refers to the distance between group $\mathrm{m}$ and $\mathrm{n}$, and $\hat{r}_{m n}$ refers to the direction among groups.

The above condition is applied under the condition of the MLFMM. In addition, when $r_{i m}+r_{n j}<r_{m n}$, group $\mathrm{m}$ and group $\mathrm{n}$ are not overlapped and neighboured, it can be met. When the MLFMM is used, laying quantity $L_{c} \geq 2$ should be met. 
In a traditional MLFMM, all $\mathrm{k}$ on Ewald ball are included during transmission. Therefore, when large-scale problems are analysed, a lot of $\hat{k}$ should be considered during numerical value analysis. In this paper, $(\theta, \phi)$ is used for describing Ewald ball, wherein, $0 \leq \theta \leq \pi, 0 \leq \phi \leq 2 \pi$. In the scope $(0, \pi)$, $\theta$ can be appropriately expressed by $L$ points. In the scope $(0,2 \pi), 2 L$ points are used for expression. The angle with coordinate axis $z$ is 0 degrees, namely $\theta=0$, the angle with coordinate axis $z$ is 180 degrees, namely, when $\theta=\pi$, all k can be expressed as follows:

$K=2 L^{2}+4$

When $D=10 \lambda, K=9,912$ under the condition of low precision requirement, $K=11,063$ during high precision requirements.

In this paper, the transmission factor $\alpha_{m n}$ is considered again in order to improve the efficiency. Equation (37) shows that transmission factor $\alpha_{m n}$ is the function related to the included angle $\cos \theta_{0}=\hat{k} \cdot \hat{r}_{m n}$ of group interval $k r_{m n}$, group size $k D$ and $\hat{k} \hat{r}_{m n}$. When two groups are separated, namely they are not overlapped, the main part of their interaction transfer factors comes from the tamper area of ray directions in two groups, namely from group $\mathrm{m}$ to group $\mathrm{n}$, only $\hat{k}$ around the function direction has strong influence on transfer factor.

In this paper, the interruption coefficient mentioned in Equation (37) is emphasised again in order to better utilise the above-mentioned ideas. The interruption coefficient is obtained after a rectangular window is used. However, other windows can also be used for interruption in the paper. The 2D condition can be regarded as an example in the paper:

$$
\alpha_{m n}\left(\hat{k} \cdot \hat{r}_{m n}\right)=\sum_{j=0}^{L} i^{j}(2 j+1) h_{j}^{(1)}\left(k r_{m n}\right) P_{j}\left(\hat{k}_{\bullet} \hat{r}_{m n}\right) \omega_{j}
$$

In the above equation (38), $\omega_{j}$ refers to the window function. When, $\omega_{j}=0$ and $j \in(L / 2, L], \omega_{j}$ is reduced with cosine function form.

The above-mentioned ray multipole method has the following though: the transfer factor $\alpha_{m n}$ is interrupted by a window function. Therefore, the value of the transfer factor $\alpha_{m n}$ around the direction from group $m$ to group $n$ can be obtained, thereby ignoring the transfer factor value of other parts. Since the transfer factor $\alpha_{m n}$ value around the direction from group $\mathrm{m}$ to group $\mathrm{n}$ is the main part affecting transfer factor. In the transfer process, the value with less influence on the transfer factor in most part is ignored in the paper after interruption through the above-mentioned window function. After the ray-propagation fast multipole method is used, the fast multipole part can obtain higher efficiency.

\subsection{Fast far-field approximation method}

It is firstly assumed that there are two points $r_{i}$ and $r_{j}$ in the calculation area. Then, it is assumed that $r_{i}$ is the field point in the mth observation group. However, $r_{j}$ is the origin of the nth source field group. Finally, $r_{m}$ and $r_{n}$ respectively represent the centres of the observation group and the origin field group. Therefore, the vector from field point to origin can be expressed as $r_{i j}=r_{i}-r_{j}=r_{i m}+r_{m n}+r_{n j}$. If group $\mathrm{m}$ and group $\mathrm{n}$ are not overlapped or adjacent, $r_{i m}+r_{n j}<r_{m n}$ can be obtained, therefore, the spherical wave equation can be expanded as follows:

$$
\frac{e^{i k\left(r_{i}-r_{j}\right)}}{r_{i}-r_{j}}=\frac{i k}{4 \pi} \int d^{2} \hat{k} e^{i k \cdot\left(r_{i m}+r_{n j}\right)} \alpha_{m n}\left(\hat{k} \cdot r_{m n}\right)
$$

In Equation (39), $\alpha_{m n}$ is defined as follows:

$$
\alpha_{m n}\left(\hat{k} \bullet \hat{r}_{m n}\right)=\sum_{l=0}^{L} i^{l}(2 l+1) h_{l}^{(1)}\left(k r_{m n}\right) P_{l}\left(\hat{k} \bullet r_{m n}\right)
$$


$L$ refers to the truncation item quantity, namely multipole modulus, $h_{l}^{(1)}(\bullet)$ refers to the first category of a Bessel function, and $P_{l}(\bullet)$ refers to a Legendre polynomial expansion.

Equation (39) and Equation (40) show that the numerical value should be estimated from many $\hat{k}$ directions during the integral. In this paper, electric distance $k\left(r_{i}-r_{j}\right)$ is given as shown in Equation (41):

$$
\begin{aligned}
& k\left|r_{i}-r_{j}\right|=k \sqrt{\left(r_{i m}+r_{m n}+r_{n j}\right) \cdot\left(r_{i m}+r_{m n}+r_{n j}\right)} \\
& =k r_{m n} \sqrt{1+2 \frac{r_{m n}}{r_{m n}^{2}} \cdot\left(r_{i m}+r_{n j}\right)+\frac{\left|r_{i m}+r_{n j}\right|^{2}}{r_{m n}^{2}}}
\end{aligned}
$$

When the distance among groups meets the following condition:

$$
\frac{r_{i m}+r_{n j}}{r_{m n}} \ll 1
$$

Equation (42) can be expanded with a Taylor series as follows:

$$
k\left|r_{i}-r_{j}\right| \approx k r_{m n}+k \hat{r_{m n}} \bullet\left(r_{i m}+r_{n j}\right)+\frac{\sin ^{2} \vartheta}{2 r_{m n}} k\left|r_{i m}+r_{n j}\right|^{2}
$$

The following condition is considered:

$$
\frac{k\left|r_{i m}+r_{n j}\right|^{2} \ll 1}{2 r_{m n}}
$$

The electric distance can be further simplified as follows:

$$
k\left|r_{i}-r_{j}\right| \approx k r_{m n}+k \hat{r_{m n}} \cdot\left(r_{i m}+r_{n j}\right)
$$

Therefore, the spherical wave equation in the far-field part can be approximated the following:

$$
\frac{e^{i k\left(r_{i}-r_{j}\right)}}{r_{i}-r_{j}} \approx \frac{i k}{4 \pi} e^{j K_{0}} \cdot\left(r_{i m}+r_{n j}\right) \alpha_{m n}^{f a r}
$$

Wherein $K_{0}=k \hat{k_{0}}$, but $\hat{k_{0}}=\hat{r_{m n}}, \alpha_{m n}^{f a r}$ refers to the far-field transmission coefficient, $\alpha_{m n}^{f a r}=4 \pi \frac{e^{i k r_{m n}}}{i k r_{m n}}$. Equation (46) shows that $k_{0}$ in only one direction is used in Equation (46).

In the paper, the above-mentioned ray-propagation fast multipole method and fast far-field approximation methods are combined and applied to finite element boundary integral method. Numerical value is analysed for different models in order to validate correctness and feasibility of procedures after addition of the above two methods.

\subsection{Example introduction and result analysis}

In order to validate the accuracy of FEBI with ray-propagation fast multipole and fast far-field approximation, in the paper, a coated missile model is analysed. The missile model is shown in Figure 1. The missile model $\mathrm{X} / \mathrm{Y} / \mathrm{Z}$ size is $0.6 \mathrm{~m} / 0.6 \mathrm{~m} / 3.75 \mathrm{~m}$. The coating material thickness is $0.05 \mathrm{~m}$.

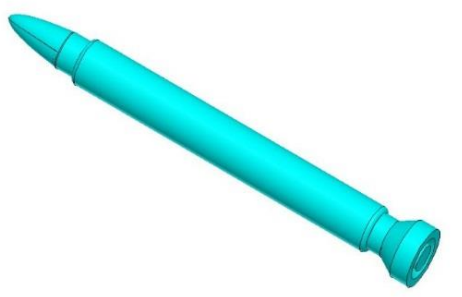

Figure 1. Coated missile model. 
The coating missile profile size is 0.04 wavelength, triangle quantity is 34250 , and tetrahedron quantity is 280002 after subdivision, and total unknown quantity is 383652 . The $\mathrm{d}$ of the coating layer material is 2.0. The incident wave frequency is $600 \mathrm{MHz}$. The incident angles of the incident wave are $\theta=180^{\circ}$ and $\varphi=180^{\circ}$. The incident wave polarisation mode is $\theta$ polarisation. The layering quantity of the boundary integral part of the MLFMM is 4 layers.

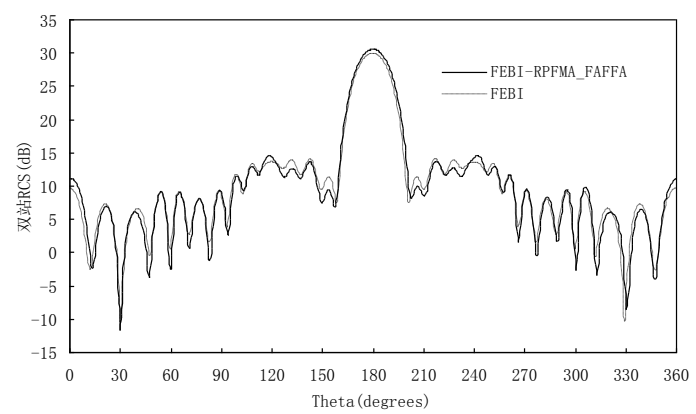

Figure 2. Coated missile double station $\operatorname{RCS}\left(\varphi=0^{\circ}\right)$.

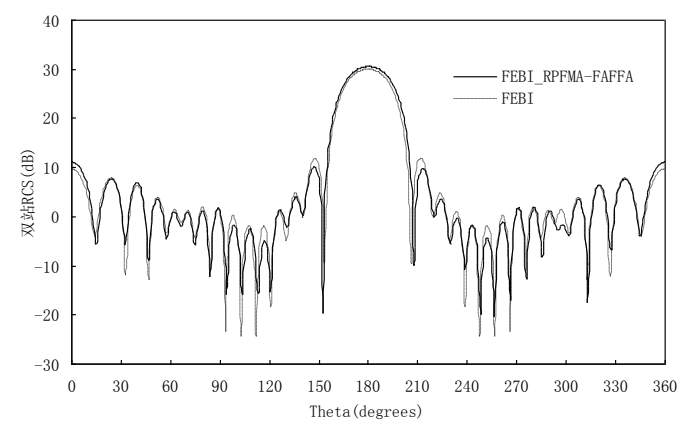

Figure 3. Coated missile double station $\operatorname{RCS}\left(\varphi=90^{\circ}\right)$.

Table 1. Comparison of iteration steps and iteration time

\begin{tabular}{|c|c|c|}
\hline & Iteration steps & Iteration time \\
\hline FEBI & 73 & $171 \mathrm{~s}$ \\
\hline FEBI-RPFMA-FAFFA & 73 & $165 \mathrm{~s}$ \\
\hline
\end{tabular}

In this paper, a coated missile column model is tested in order to test the memory saving condition of the procedure calculation after ray-propagation fast multipole and fast far-field approximation are added. The model column is $8 \mathrm{~m}$ high, its radius is $0.15 \mathrm{~m}$, the coating layer thickness is $0.05 \mathrm{~m}$, and the dielectric constant of coating material is 1.2. The profile size of the coated column is 0.02 wavelength. The incident wave frequency is $1.0 \mathrm{GHz}$. The incident wave incident angles are $\theta=180^{\circ}$ and $\varphi=180^{\circ}$. The incident wave polarisation mode is $\theta$ polarisation. Convergence precision is $1.0 \mathrm{e}-3$. Source program iteration was divided into 123 steps. After fast far-field approximation was added, there were 124 iteration steps. In the boundary integral part, the MLFMM is divided into six layers. In this paper, ray-propagation fast multipole method and fast far-field approximation method were used aiming at different layers of fast multipole aiming at the above coated column example. The memory required for the calculations is shown in Table 2.

Table 2. Comparison of memory and iteration of different group numbers of FEBI and FEBI-RPFMA-FAFFA

\begin{tabular}{|c|c|c|c|}
\hline & Far-field memory (MB) & Iteration steps & Iteration time \\
\hline FEBI & 764 & 21 & $239 \mathrm{~s}$ \\
\hline FEBI-RPFMA-FAFFA (FMM four-layer) & 657 & 21 & $223 \mathrm{~s}$ \\
\hline FEBI-RPFMA-FAFFA (FMM five-layer) & 563 & 22 & $213 \mathrm{~s}$ \\
\hline FEBI-RPFMA-FAFFA (FMM six-layer) & 489 & 23 & $203 \mathrm{~s}$ \\
\hline
\end{tabular}

In this paper, Ray-Propagation Fast Multipole method (RPFMA) and FAst Far-Field Approximation (FAFFA) method are also applied to the boundary integral part of a finite element boundary integral 
method. Analysis of the numerical values of the above different models shows that the procedure efficiency is improved after the addition of ray-propagation fast multipole method and fast far-field approximation method, thereby reducing the memory required for the calculation on the one hand, and reducing the calculation time on the other. However, the results from the calculations are well matched with the results without the addition of the two methods. The feasibility of the above two methods used in the boundary cloud boundary integral method is described.

\section{Conclusion}

In the paper, the basic principles of ray-propagation fast multipole method and fast far-field approximation method are analysed and discussed. After the two methods are combined, they can be used for the boundary integral part of a finite element boundary integral method. Analysis of missiles and other models shows that the memory obtained through the calculation is reduced in the far-field part of the boundary integral. The memory consumption for the calculation is reduced, thereby high efficiency of the procedure after the addition of the ray-propagation fast multipole method and the fast far-field approximation method in the finite element boundary integral method is proved.

\section{References}

[1] Graglia, R. D., Peterson, A. F., Petrini, P., et al. (2015). Hierarchical vector bases for 3D problems. Usnc-Ursi Radio Science Meeting.

[2] Ji, Yun. (2000). Development and applications of a hybrid finite-element method/ method -of-moments (FEM/MoM) tool to model electromagnetic compatibility and signal integrity problems in printed circuit boards.

[3] Patel, U. R. and Triverio, P. (2010). MoM-SO: A Complete method for computing the impedance of cable systems including skin, proximity, and ground return effects. IEEE Transactions on Power Delivery, 30(5), 2110-2118.

[4] Reddy, V. S. and Garg R. (2015). Finite Difference Time Domain (FDTD) analysis of microwave circuits-A review with examples. Iete Journal of Research, 45(1), 3-20.

[5] Ren Y., Liu, Q. H. and Chen, Y. P. (2016). A hybrid FEM/MoM method for 3-D electromagnetic scattering in layered medium. IEEE Transactions on Antennas \& Propagation, 64(8), p. 3487-3495.

[6] Umashankar, K., Taflove, A., and Rao, S. M. (1986). Electromagnetic scattering by arbitrary shaped three-dimensional homogeneous lossy dielectric objects. IEEE Transactions on Antennas \& Propagation, 34(6),758-766.

[7] Wang, Y., and Langdon, S. (2010). Design of wave ports in FDTD and its application to microwave circuits and antennas. Antennas \& Propagation Society International Symposium IEEE, $1-4$.

[8] Xu, G., Chen, X., Zheng, Z., et al. (2015). A hybrid FDTD-SPICE method for the analysis of microwave circuits. International Journal of Applied Electromagnetics \& Mechanics, 49(1), 79-90.

[9] Yang, Z. and Tan, E. (2015). A mircostrip circuit tool kit app with FDTD analysis including lumped elements. IEEE Microwave Magazine, 16(1), 74-80.

[10] Yla-Oijala, P. and Taskinen, M. (2005). Application of combined field integral equation for electromagnetic scattering by dielectric and composite objects. IEEE Transactions on Antennas \& Propagation, 53(3), 1168-1173. 